\title{
Hedgehog directly controls initiation and propagation of retinal differentiation in the D rosophila eye
}

\author{
María Domínguez ${ }^{1,2,3}$ and Emst Hafen ${ }^{1}$ \\ ${ }^{1}$ Zoological Institute, University of Zürich, CH-8057 Zürich, Switzerland
}

\begin{abstract}
Patteming of the compound eye begins at the posterior edge of the eye imaginal disc and progresses anteriorly toward the disc margin. The advancing front of ommatidial differentiation is marked by the morphogenetic furrow (MF). Here we show by clonal analysis that Hedgehog (Hh), secreted from two distinct populations of cells has two distinct functions: It was well documented that Hh expression in the differentiating photoreceptor cells drives the morphogenetic furrow. Now we show that, in addition, Hh, secreted from cells at the posterior disc margin, is absol utely required for the initiation of patteming and predisposes ommatidial precursor cells to enter ommatidial assembly later. These two functions of $\mathrm{Hh}$ in eye patterning are similar to the biphasic requirement for Sonic Hh in patteming of the ventral neural tube in vertebrates. We show further that Hh induces ommatidial development in the absence of its secondary signals Wingless (Wg) and Dpp and that the primary function of Dpp in MF initiation is the repression of $\mathbf{w g}$, which prevents ommatidial differentiation. Our results show that the regulatory relationships between $\mathrm{Hh}$, Dpp, and $\mathrm{Wg}$ in the eye are similar to those found in other imaginal discs such as the leg disc despite obvious differences in their modes of development.
\end{abstract}

[Key Words: Drosophila eye; morphogenetic furrow initiation; hedgehog signaling]

Received August 18, 1997; revised version accepted September 25, 1997.

M embers of the Hedgehog $(\mathrm{Hh})$ family of proteins have been implicated in patterning of multiple tissues during embryogenesis. These include the neural tube, limbs, bone, sexual organs, and eyes in vertebrates and invertebrates (for review, see Hammerschmidt et al. 1997). The molecular basis of $\mathrm{Hh}$ signaling is best understood in its role in patterning the anteroposterior axis of the embryonic segments and the appendages of Drosophila. Hh appears to control patterning indirectly by locally inducing the expression of secondary signaling molecules that can act at a distance (for review, see Hammerschmidt et al. 1997). In the embryo and the ventral part of the leg imaginal disc, $\mathrm{Hh}$ induces the expression of wingless (wg) in anterior cells at the anteroposterior compartment boundary (Forbes et al. 1993; Basler and Struhl 1994; Tabata et al. 1995; $\mathrm{Ng}$ et al. 1996). In the dorsal part of the leg disc and in the wing disc, Hh induces Dpp (Basler and Struhl 1994; Tabata et al. 1995). Recent experiments have suggested that there is not just a simple linear relationship between $\mathrm{Hh}$ and its two secondary signals, Dpp and Wg. On one hand, Dpp and Wg not only control different cell fates in response to $\mathrm{Hh}$ signaling, but they

${ }^{2}$ Present address: Medical Research Council Laboratory of Molecular Biology, Cambridge CB2 2QH, UK.

${ }^{3}$ Corresponding author.

E-MAIL mdl@mrc-Imb.cam.ac.uk; FAX 441223412142. also mutually repress each others transcription (Brook and Cohen 1996; Jiang and Struhl 1996; Johnston and Schubiger 1996; Morimura et al. 1996; Peton and Hoffmann 1996; Heslip et al. 1997). It is therefore possible that Dpp specifies cell fate not only by directly acting on target cells in a concentration dependent manner but also indirectly by repressing wg. On the other hand, $\mathrm{Hh}$ may also control cell fate directly independent of Dpp and Wg. In experiments in which $\mathrm{Hh}$ and Dpp are ectopically expressed in the developing wing, $\mathrm{Hh}$ but not Dpp is sufficient to induce sensory structures normally found in anterior cells near the compartment boundary (Gómez-Skarmeta and Modolell 1996; Mullor et al. 1997). Furthermore, Hh may also act directly in patterning of the dorsal epidermis (Bokor and DiN ardo 1994). Because Dpp is required earlier for the establishment of the dorsoventral axis of the embryo, however, it is unclear whether $\mathrm{Hh}$ acts al one or in combination with $\mathrm{Dpp}$ to specify cell identities.

Two independent roles for $\mathrm{Hh}$ and $\mathrm{Dpp}$ have been de scribed in patterning the Drosophila compound eye (for review, see Heberlein and M oses 1995). In contrast to the wing and legs, patterning of the devel oping eye occurs by a lineage-independent mechanism and is closely linked to cellular differentiation (for review, see Wolff and Ready 1993; Bonini and Choi 1995). Pattern formation is initiated at the late second/ early third instar larval stage at posterior part of the eye disc and spreads anteriorly by the movement of the morphogenetic furrow (MF). The 
sequential induction of the $\mathrm{MF}$ is driven by $\mathrm{Hh}$, which is secreted from the differentiating ommatidial cells (for review, see Heberlein and Moses 1995). Hh induces ommatidial assembly in more anterior cells, some of which, in turn, become $\mathrm{Hh}$ secreting cells and, thus, the MF advances. Although dpp expression is also induced by $\mathrm{Hh}$ in the MF (Heberlein et al. 1993), Dpp signaling appears to be dispensable in the wild type for ommatidial assembly and MF propagation (Burke and Basler 1996; Wiersdorff et al. 1996; Chanut and Heberlein 1997; Peton et al. 1997). A reciprocal requirement for $\mathrm{Hh}$ and $\mathrm{Dpp}$ was observed in the initiation of the MF at the posterior end of the eye disc and in its continuous reinitiation along the lateral margins. MF initiation is blocked in clones of mutant cells lacking the Dpp-receptor Thick veins (Tkv) or the Dpp-downstream protein Mothers against dpp ( $\mathrm{Mad}$ ) that include the posterior and lateral disc margins (Burke and Basler 1996; Wiersdorff et al. 1996). On the basis of three observations, it has been concluded that $\mathrm{Hh}$ is not involved in MF initiation from the posterior margin nor in its reinitiation from the lateral margins (Heberlein et al. 1993; Ma et al. 1993; Jarman et al. 1995). Firstly, the M F initiates normally but arrests prematurely in $\mathrm{hh}^{1}$ eye discs (Heberlein et al. 1993). $\mathrm{hh}^{1}$ is a partial loss-of-function allele that specifically impairs hh function in the developing eye (Lindsley and Zimm 1992). Second, when $\mathrm{hh}^{\text {ts2 }}$ larvae were grown at the permissi ve temperature until early third instar and then shifted to the restrictive temperature, progression in the center of the disc, but not initiation of the M F, was blocked (M a et al. 1993). Third, the M F still initiates and propagates a short distance before it stops in eye discs mutant for atonal (ato), where ommatidial development and, hence, hh expression in the ommatidial cells is completely abolished (see references in Heberlein et al. 1995).

Here we have reassessed the requirement for $\mathrm{Hh}$ in patterning the eye disc and its relation to the function of $\mathrm{Dpp}$ and $\mathrm{Wg}$, (1) by examining the effects of complete loss of hh function in somatic clones, (2) by examining the expression of hh in relation to early cell markers for the MF, and (3) by examining the consequences of constitutive activation of the $\mathrm{Hh}$ signal transduction pathway in cells unable to produce Dpp and Wg. In contrast to previous reports, our results show an absolute requirement for $\mathrm{Hh}$ in the initiation of patterning in the eye. In addition, we show that $\mathrm{Hh}$ and $\mathrm{Dpp}$ signaling pathways contribute to eye patterning independently. Hh acts directly in the control of the initiation and propagation of the M F. In contrast, D pp is required indirectly to prevent marginal cells that receive the $\mathrm{Hh}$ signal from expressing wg. This repression is important because activation of wgin the $\mathrm{Hh}$ receiving cells prevents MF induction by $\mathrm{Hh}$.

\section{Results}

$\mathrm{Hh}$ is required both for initiation and progression of the morphogenetic furrow

We have examined the effects of loss of hh function in the eye imaginal disc. To do this, we generated clones of cells mutant for the null allele, $\mathrm{hh}^{\mathrm{AC}}$ (Ma et al. 1993) by use of the FRT-FLP technique (Xu and Rubin 1993). Two classes of hh mutant clones were observed in the eye disc. The first class consists of clones that are situated entirely within the eye field. In these clones, MF propagation and ommatidial differentiation, assessed by the expression of the neuronal marker Elav, is normal (Fig. 1). Only in the center of large cl ones is the progression of the $M F$ retarded relative to the adjacent tissue (Fig. $1 A, B)$. In addition, the expression level of the proneural gene ato, which is required for the initiation of neuronal differentiation in the MF (Jarman et al. 1994), is reduced in the center of the clone (Fig. 1A). These results are consistent with the phenotype described for hh clones in the adult eye (Heberlein et al. 1993; Ma et al. 1993), which showed that photoreceptor differentiation occurs normally in the mutant cells and that only in the center of I arge clones are aberrant ommatidia found. Because neuronal differentiation and M F propagation can proceed normally through and beyond mutant hh clones situated entirely within the eye field, it appears that $\mathrm{Hh}$ secreted from the neighboring wild-type ommatidia rescues, in a nonautonomous manner, the loss of hh. On the basis of the rescue of mutant ommatidial units, we estimate that adequately high levels of $\mathrm{Hh}$ protein reach hh mutant cells across a distance of about three ommatidial clusters from the boundary with $\mathrm{hh}^{+}$cells.

Clones of the second class include some of the lateral or posterior margin of the eye disc (Fig. 2). In these clones, no neuronal differentiation is observed (Fig. $2 A, C, E-G)$, except close to the clone boundary, where single hh mutant ommatidia can be rescued (see Fig. 2A and arrowheads in $2 \mathrm{C}$ ). The changes in cell shape that precede and accompany neuronal differentiation are al so absent in these marginal clones (Fig. 2E-G). These results show an essential early role of $\mathrm{Hh}$ in the initiation of the MF, in addition to its role in MF propagation. Interestingly, there is a significant difference in the re quirements for $\mathrm{Hh}$ at the disc margins as opposed to the internal eye field, as inferred by the different degree of nonautonomy between marginal (Fig. 2) and internal (Fig. 1) clones.

The analysis of hh in clones in the adult eye (Heberl ein et al. 1993; Ma et al. 1993) showed that, whereas the majority of clones developed normal ommatidial structures as a result of the nonautonomous rescue by neighboring $\mathrm{hh}^{+}$cells, a small fraction of clones caused gross abnormalities, including the absence of large portions of the eye (Heberlein et al. 1993). This class of clones may correspond to the class of marginal clones.

Blocking the reception of Dpp in Mad or tkv mutant clones at the margin is often associated with the induction of an ectopic eye field adjacent to the clone (Wiersdorff et al. 1996 and Fig. 2H). In contrast, we have never observed similar reorganizations of eye discs containing hh mutant clones (see also $M$ aterials and $M$ ethods). Moreover, Mad mutant cells that fail to form eye structures develop dorsal head structures instead (Wiersdorff et al. 1996). The formation of dorsal head structures can be attributed to the gain of wg expression 
Figure 1. Effects of loss of hh function during M F propagation. In all images, eye discs are oriented dorsal to the right and anterior up. Third instar larval eye discs containing hh internal clones double stained with antibodies against $\beta$-galactosi dase (red) and the proneural protein Ato (green in A) or the neuronal marker Elav (green in B). Single and superimposed confocal images are shown side by side. The homozygous mutant cells (hh/hh) are marked by the absence of lacZ staining. (A) The clone spans the M F and causes a reduction in the levels of $A$ to protein (arrowhead) in the MF. N ote the slightly bent line of ato expression, suggesting that the M F has progressed more slowly in the region with limited $\mathrm{Hh}$ signal. (B) The disc contains two fused hh clones. The anterior clone (arrowhead) spans the M F at the time of dissection. The second clone is located posterior to the MF. Examination throughout the depth of the two clones
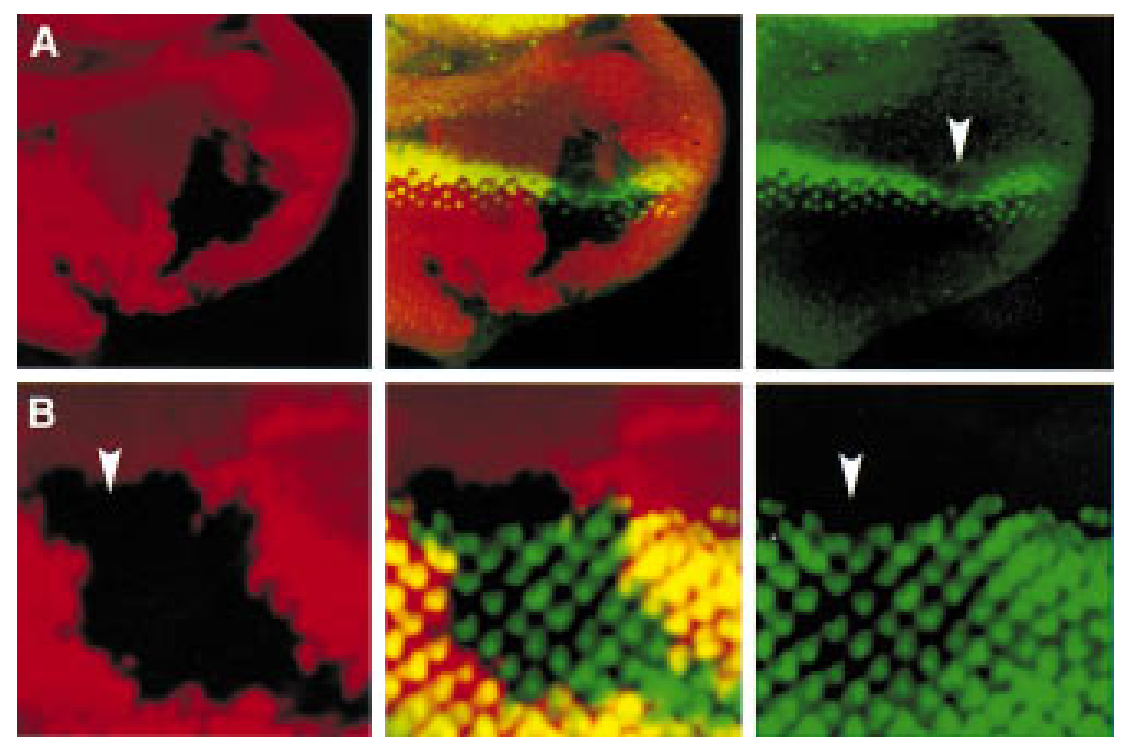

shows that neuronal differentiation, assessed by Elav expression (green), has proceeded normally within the mutant tissue. On the basis of the rescue of mutant ommatidial units, we estimate that adequately high levels of Hh protein reach hh mutant cells across a distance of about three ommatidial clusters from the boundary with $\mathrm{hh}^{+}$cells.

in the posterior mutant Mad cells (Wiersdorff et al. 1996). In contrast, marginal hh clones lead to formation of naked cuticle in the adult eye (data not shown; $\mathrm{He}$ berlein et al. 1993). We have not observed ectopic wg expression in posterior marginal hh clones at third instar larval stage (data not shown).

\section{Early expression of hh, ptc, and dpp in the eye disc}

The results from our clonal analysis of hh show an essential role of $\mathrm{Hh}$ in the initiation of the MF from the posterior margin of the disc, which is in conflict with a previous observation that $\mathrm{hh}$ is only expressed in the differentiating photoreceptor cells after the M F has been initiated ( $M$ a et al. 1993). Therefore, we re-examined the expression of hh at the time of MF initiation in the late second and early third larval instar stages. The expression of the patched (ptc) gene, which is activated in response to Hh signaling (Capdevila et al. 1994; Heberlein et al. 1995; T abata et al. 1995; Strutt and M lodzik 1996), was also examined at the second and third instar larval stage. To monitor hh expression, we used the enhancertrap line $\mathrm{hh}^{\mathrm{P} 30}$. This line contains a $\mathrm{P}(\mathrm{lacZ}$ ) insertion in the hh locus and reproduces the pattern of the endogenous gene (Lee et al. 1992; Ma et al. 1993). ptc expression was visualized by use of the ptc ${ }^{\text {AT96 }}$ enhancer-trap line (see Materials and M ethods). The onset of MF initiation was visualized by use of an antibody against the Ato protein (Jarman et al. 1994), which we found is induced shortly before cells enter the MF, assessed by changes in cell shape visualized by the anti-Arm antibody (Fig. 3A). hh-lacZ is al ready expressed in the late second/early third instar larval disc along the posterior and dorso-lateral margins (Fig. 3B-D, G). hh-lacZ expres- sion is weak and becomes stronger in cells just posterior to the first A to positive cells (Fig. 3C). To substantiate that this early expression of $\beta$-galactosi dase in the $\mathrm{hh}^{\mathrm{P} 30}$ line al ong the disc margin is rel ated to MF induction, we have examined hh-lacZ and ato expression in slightly more mature discs, when the MF has moved anteriorly. As shown in Figure 3D, the advance of the MF is closely associated to the anterior expansion of hh-lacZ. The hh expressing cells lie between discrete Ato-positive cells, which correspond to the presumptive R8 photoreceptor cells (Fig. 3D). It was shown that hh-lacZ expression in the devel oping photoreceptor cells starts several ommatidial rows posterior to the M F as ommatidial cells initiate neuronal differentiation (Heberlein et al. 1993; $\mathrm{Ma}$ et al. 1993). At the stage shown in Figure 3D, the neuronal antigen recognized by the Elav antibody is not yet expressed in the differentiating photoreceptor cells (not shown). By this criterion, this early expression of hh precedes neuronal differentiation by several hours and, hence, cannot be dependent on it. Furthermore, whereas the later expression of hh is restricted to a subpopulation of developing photoreceptors ( $\mathrm{M}$ a et al. 1993), the early expression of hh is observed in all cells of the posterior margin that themselves will not contribute to the eye field proper, and also in the posterior ommatidial precursors prior to their recruitment. This early expression of hh may explain why the MF still initiates in ato mutant discs in which neuronal differentiation (Jarman et al. 1994, 1995) and, hence, hh expression in differentiating ommatidial cells is prevented. ptc expression, which reflects hh activity, is also present in the young eye disc (Fig. $3 \mathrm{H}$ ), supporting the vi ew that the $\mathrm{Hh}$ signaling pathway is al ready active at this stage.

The signaling molecules Dpp and Wg, which are also 

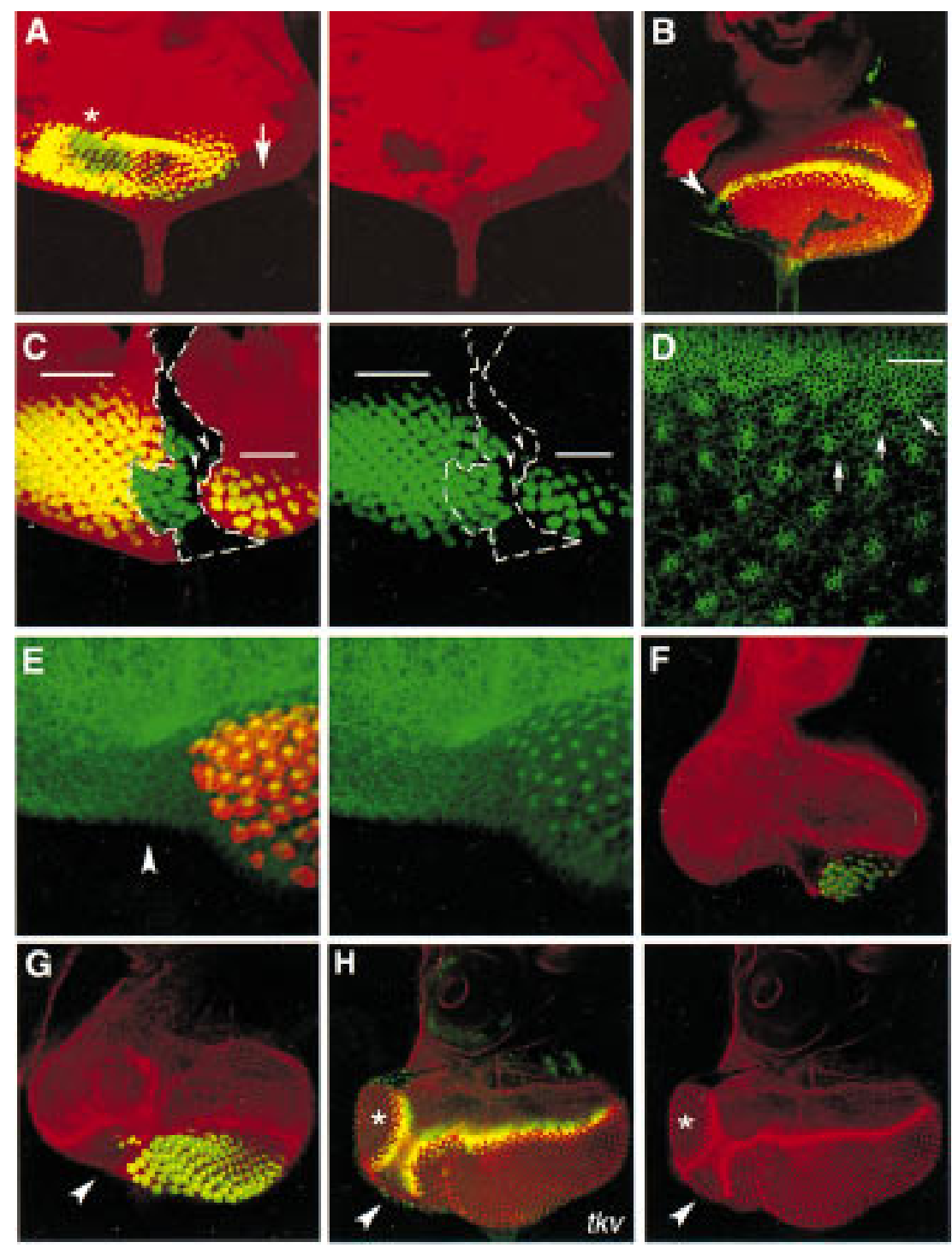

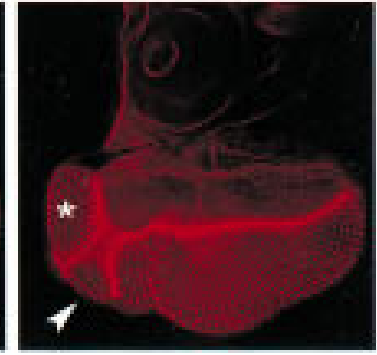

Figure 2. Role of hh in the initiation of the MF. (A-C and E-G) Mosaic eye discs carrying hh clones that include the disc margin. $(A-C)$ hh clones are marked by the absence of lacZ (red) staining. ( $E-G$ ) hh mutant tissue is marked indirectly by the absence of Elav (green). (A) The disc contains several hh clones. Single-channel image of the armlacZ staining to visualize the position of the clones is shown in the right panel. N euronal differentiation, assessed by Elav expression, is normal in the internal hh clone (asterisk), whereas it is blocked in the marginal hh clone (arrow). The arrow points to the center of the marginal clone and also where marginal ommatidia are missing. (B) The expression of ato (green) is al so affected in marginal hh clones. N ote that only cells in close proximity to the wild-type border of the clone have detectable Ato protein levels (arrowhead). (C) A large hh clone (outlined in white) that runs al ong the center of the disc and spans half of the posterior eye margin. The single channel image of the Elav (green) is shown in the right panel. N euronal differentiation is abolished in the area where the posterior margin is mutant for hh. In contrast, the $\mathrm{hh}^{+}$margin initiated the $\mathrm{MF}$, which progressed normally through an internal hh mutant area (note the presence of green ommatidia). Only a few mutant ommatidia (arrowheads) are rescued adjacent to the borders of the marginal part of the clone. The bars in the image show the approximate position of the MF. (D) A portion of a wildtype disc stained with a monoclonal antibody raised against Arm (see also Materials and $M$ ethods) to show changes in cell shape associated with cells in the MF (white line) and cells in the ommatidial clusters (some of them are marked with arrows). The image shows an apical focal plane. (E-G) Eye discs carrying marginal hh clones that span half of the posterior margin. hh cells marked by the absence of Elav staining (red in E, and green in F and G) do not constrict apically and fail to assemble into ommatidial clusters, as assessed by the Arm staining (green in E and red in F and G). The disc in E was dissected at the mid third instar stage to confirm that failure to initiate ommatidial differentiation is not an indirect effect of loss of hh in the ommatidial clusters. The Arm staining is in red, and the Elav in green. The clones in F and G were induced in a Minute background (see $M$ aterials and $M$ ethods). (H) M osaic eye disc carrying a tkv clone al so induced in a Minute background. The uniform Arm staining (red) and the absence of ato expression (green) in the region marked by the arrowhead indicates that this region lacks tkv. Adjacent to this area, a supernumerary eye field (asterisk) has formed in the ventral region of the eye disc (see $M$ aterials and $M$ ethods). The ectopic eye develops an equator as in the endogenous eye, indicating that loss of Dpp reception, but not loss of hh (cf. E-G with H), results in the reprogramming of positional information in the marginal cells to initiate an MF in an ectopic position.

required for patterning of the eye, are al ready expressed in the late second, early third instar eye disc. wg-lac Z is expressed at the disc margin before the onset of ato expression (not shown). At these early stages, wg-lacZ expression is confined to the most anterior margin of the disc, from which most of the head capsule develops (Fig. 3F). dpp is expressed al ong the posterior and lateral margin of the disc (Masucci et al. 1990; Blackman et al. 1991; and Fig. 3E). We consistently observe a slight temporal difference, however, in the appearance of hh and dpp expression at the posterior margin of young discs. Whereas hh is strongly expressed at the posterior margin of the disc prior to ato expression, dpp expression in the BS3.0 line appears at the time of ato expression (Fig. 3 cf. $\mathrm{C}$ and $\mathrm{E})$.

Relationship between $\mathrm{Hh}, \mathrm{Dpp}$, and Wg signaling in the initiation of the MF

The results presented here indicate that $\mathrm{Hh}$ activity is 
Figure 3. Early pattern of hh expression in the second and early third instar eye disc. Anterior is up in all panels. (A) The first prominent accumulation of A to protein (red, left panel) in the eye disc marks the onset of the MF, as assessed by Arm staining (green, right panel). The middle panel shows the combined image. (B-F) Confocal images of increasingly older discs stained with anti-A to antibody (green) and $\beta$-gal actosi dase (red) to detect lacZ expression in the $h^{\mathrm{P} 30}(\mathrm{~B}-\mathrm{D})$, in the dpp-BS3.0 line (E) or in a wglacZ line (F). The expression of hh occurs in a line of cells al ong the posterior and dorsal margin of the eye disc prior to the initiation of the M F (B). This expression is el evated at the posterior most edge of the disc at the time of M F induction when the first Ato positive cells (green) are detectable (C). The right panel shows only hh-lacZ expression in the same disc. (D) An older eye disc, in which the MF has progressed three ommatidial rows (indicated by arrows). The expression of hh (arrow) has expanded anteriorly to the region of the presumptive dorsal head (dh) and is located posterior to the band of continuous Ato expression (arrowhead). The hh-lacZ-positive cells (red, arrow) are in between the single Ato-positive cells, which correspond to R8 precursor cells. Double staining with anti-A to and anti-Elav showed no expression of Elav at this early stage (not shown). (E) dpp-lacZ expression was examined in relation to the early expression of ato. $\mathrm{N}$ ote that the induction of the MF is not synchronous in the two eye discs of the same larva. The disc that shows no detectable accumulation of Ato protein also has weaker expression of dpp at the most posterior edge of the disc (arrow). The dpp-lacZ expression in this region becomes detectable at the time of ato induction, but dpp expression is still weaker and discontinuous (arrow) in this region compared with its expression in the lateral margins. (F) The disc is of the same age as that shown in D. wg-lacZ expression is confined to the anterior part of the disc. N ote that the prominent wg-lacZ expression in the presumptive dorsal head region (dh) overlaps at this stage with hh-lacZ expression. (G-H) Y oung eye discs of $\mathrm{hh}^{\mathrm{P} 30}(\mathrm{G})$ and $\operatorname{ptc}^{\mathrm{AT} 96}(\mathrm{H})$ larvae stained with X-gal (blue). $\mathrm{N}$ ote that the stripe of ptc-lac $\mathrm{Z}$ is broader than the stripe of hh-lacZ consistent with the notion that secreted $\mathrm{Hh}$ can induce gene expression in cells farther from the hhexpressing cells. The discrepancy between the time and place of expression of $\beta$-galactosidase in the $h^{\mathrm{P} 30}$ line shown here and the previous reported hh RNA distribution is most likely attributed to the different age of the eye discs used in these two studies. Whereas Ma et al.
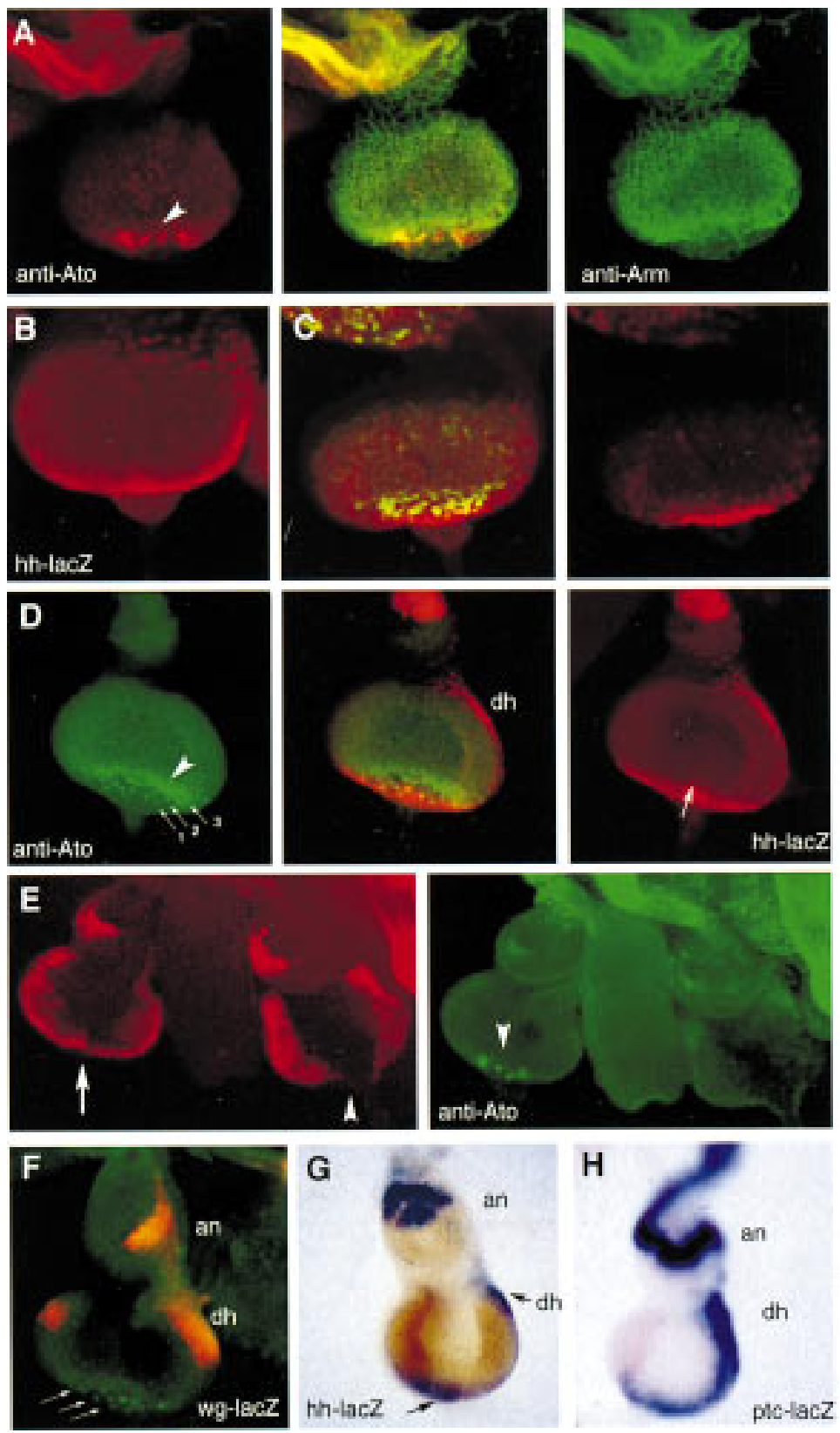

plc-lacz

(1993) analyzed hh RN A distribution only in the third instar disc, we see hh-lacZ expression at the disc margin al ready in second instar disc. We used a cell marker (anti-A to) and a morphol ogical marker (anti-Arm) to assess directly the age of the eye discs. A bbreviations: an, antennal disc; dh, presumptive dorsal head.

required for the initiation of the $\mathrm{MF}$ and are consistent with previous reports showing that activation of $\mathrm{Hh}$ signaling in cells anterior to the M F is sufficient to induce ectopic M Fs (Heberlein et al. 1995; Ma and M oses 1995; Pan and Rubin 1995; Strutt and Mlodzik 1995; Strutt et al. 1995; Wehrli and Tomlinson 1995; Pignoni and Zipursky 1997). Because Wg and D pp are al so implicated in the control of MF initiation-Wg prevents and Dpp promotes initiation-we wondered what the relationship between $\mathrm{Hh}, \mathrm{Wg}$, and Dpp in this process was. We tested whether $\mathrm{Hh}$ alone is sufficient for this process or whether it acts indi rectly via the secondary signals Dpp or Wg. The protein kinase A (Pka) acts downstream in the Hh signaling pathway and loss of pka activity mimics the reception of the $\mathrm{Hh}$ signal (Jiang and Struhl 1995; Lepage et al. 1995; Li et al. 1995; Pan and Rubin 1995; Strutt et al. 1995). Loss of pka function, like ectopic hh expression (Heberlein et al. 1995; Pignoni and Zipursky 1997), in anterior cells induces the formation of ectopic M Fs (Pan and Rubin 1995; Strutt et al. 1995). Like ectopic hh expression (Heberlein et al. 1995; Pignoni and Zipursky 1997), loss of pka function activates dpp ex- 
pression in cells within the eye field (Pan and Rubin 1995; Strutt et al. 1995). We tested whether loss of pka also results in ectopic expression of $\mathrm{wg}$ in any region of the eye disc. As shown in Figure 4A, loss of pka function also induces wg expression in the anterior part of the eye and in a domain in the antennal disc (Fig. 4A). N ext, we tested whether activation of the $\mathrm{Hh}$ signaling pathway by loss of pka function is sufficient to induce ectopic MFs in the absence of dpp and wg function. To do this, we generated clones of cells triple mutant for pka, dpp, and wg. The expression of ato was used as an early marker for M F initiation. Clones of cells triple mutant for dpp, wg, and pka induce autonomously ectopic ex- pression of ato (Fig. 4B,C). Triple mutant cells located near to or spanning the endogenous MF cause ectopic MFs and the acceleration of the endogenous MF (Fig. 4B,C), as was descri bed for pka single mutant cl ones (Pan and Rubin 1995; Strutt et al. 1995). In addition, we find that dpp wg pka clones located far from the endogenous $M F$, including the anterior margin of the eye disc, still induce ectopic expression of ato at levels comparable with cells in the MF (not shown). The ectopic eyes induced by mi sexpression of dpp are normally formed from the anterior margin of the disc (Heberlein et al. 1995; Pignoni and Zipursky 1997) and induction of ectopic eyes is associated with removal of wg expressi on (Heber-
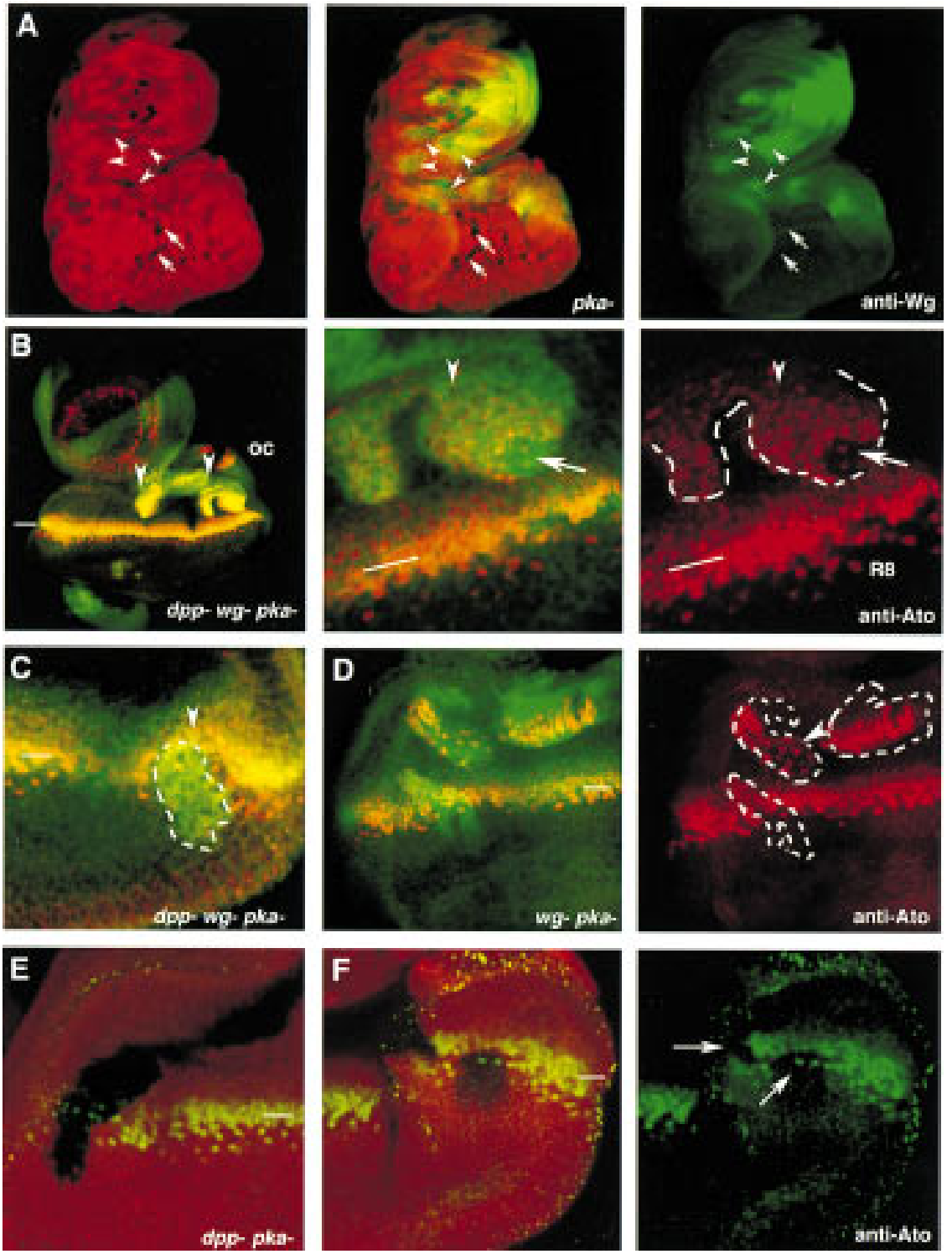

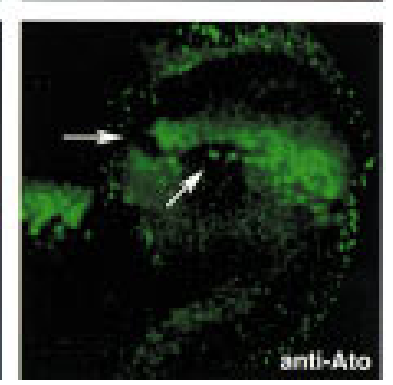

Figure 4. Constitutive activation of the Hh signaling pathway, in the absence of wg and dpp activities, activates the expression of the proneural gene ato and causes ectopic MFs. (A) A third instar larval eye disc stained with anti-wg (green) and carrying multiple pka ${ }^{\mathrm{DCO}-\mathrm{B3}}$ clones marked by the absence of arm-lacZ (red). The arrows point to eye internal single pka ${ }^{\mathrm{DCO}-\mathrm{B} 3}$ clones that do not activate wg expression. The arrowheads point to clones where wg expression is activated in response to removal of pka. $(B, C)$ Eye discs carrying $\mathrm{dpp}^{\mathrm{H} 61}$ wg ${ }^{\mathrm{CX} 4} \mathrm{pka}^{\mathrm{DCO}-\mathrm{B} 3}$, which are marked by the increased accumulation of $\mathrm{Ci}$ occurring in the pka mutant cells (Johnson et al. 1995; M. Domínguez and E. Hafen unpubl.). The dpp ${ }^{d 12}$ pkaDCO-B3 clones are marked by the absence of armlacZ staining. (B) A large $\mathrm{dpp}^{\mathrm{H} 61} \mathrm{wg}$ ${ }^{\mathrm{C} \times 4} \mathrm{pka}^{\mathrm{DCO}-\mathrm{B} 3}$ triple mutant clone (arrowheads) located in the anterior part of the eye disc. ato expression is induced autonomously in the mutant cells. A detail of the posterior part of the clone is shown in the middle panel. The right panel shows a single image of ato expression in the posterior part of the clone (outlined in white) and ato expression in the endogenous MF (indicated with the bar). In the MF (white line), the uniform levels of Ato protein evolve into discrete single Ato-positive cells, the R8 photoreceptor cells. $\mathrm{N}$ ote that most cells in the clone have uniform levels of Ato (arrowhead) as those found in the endogenous MF. Some of the mutant cells (arrow) have initiated ommatidial development, as inferred by the presence of regularly spaced Ato-positive cells. (C) A $\mathrm{dpp}^{\mathrm{H} 61}$ wg ${ }^{\mathrm{CX} 4} \mathrm{pka}^{\mathrm{DCO}-\mathrm{B} 3}$ clone located posterior to the MF at the time of dissection. The clone caused an acceleration of the endogenous M F (arrowhead). (D) Aneye disc carrying three wg ${ }^{\mathrm{CX} 4} \mathrm{pka}^{\mathrm{DCO}-\mathrm{B3}} \mathrm{mu}$

tant clones. N ote the ectopic activation of ato expression even in the clones located far from the endogenous M F. The arrowhead points to some mutant cells where ectopic expression of ato is in separated singular cells. (E,F) Two eye discs carrying dpp ${ }^{\mathrm{d} 12}$ pka $^{\mathrm{DCO}-\mathrm{B} 3}$ mutant clones. N ote that ato expression (green) is not induced in these clones, which are marked by the absence of arm-lacZ staining (red). The clones in $\mathrm{F}$ span the endogenous M F and have caused a reduction in the levels of expression of ato (arrowheads). (oc) Occellar region. The position of the endogenous MF is indicated by white bars. 
Iein et al. 1995; Pignoni and Zipursky 1997). Our results of the triple mutant clones suggest a dispensable role for Dpp in the initiation of M Fs and, thus, are in contrast to the report of Wiersdorff et al. (1996) that Dpp signaling via the $M$ ad protein is required to initiate M Fs from pka cells. One explanation for the difference between pka $\mathrm{Mad}$ and dpp wg pka clones could be the failure to activate wg expression in the latter. To test whether the loss of $w g$, rather than the gain of dpp, is necessary for the formation of ectopic MFs, we compared the triple mutant clones with clones double mutant for wg pka (Fig. 4D) or dpp pka (Fig. 4E-F). Whereas wg pka clones located in the anterior part of the disc behave as triple clones, dpp pka mutant cells, like pka Mad mutant cells (Wiersdorff et al. 1996) are unable to activate ato (Fig. $4 \mathrm{E})$. These results indicate that, at least in the lateral and anterior region of the disc, in the absence of $\mathrm{Wg}$, Dpp is di spensable for the initiation of a MF by the Hh signaling pathway. Posterior triple mutant clones, as single mutant pka clones (Strutt et al. 1995; Pan and Rubin 1995), showed normal retinal differentiation (not shown). Although we cannot exclude the possibility that neighboring $\mathrm{dpp}^{+}$tissue rescues the loss of $\mathrm{dpp}$ in the posterior marginal clones, it is important to bear in mind that in $\mathrm{dpp}^{\text {blink }}$ mutants, where no dpp expression is detected in the eye disc, the MF still initiates in the center of the posterior margin (T reisman and Rubin 1995). Our results also show that activation of Wg in the Hh-receiving cells alters the competence of cells to respond to $\mathrm{Hh}$ signals.

\section{Discussion}

$\mathrm{Hh}$ acts directly on cells in the eye imaginal disk to induce ommatidial differentiation

Our results show an essential and direct function of $\mathrm{Hh}$ in the initiation and propagation of the MF. Loss of hh from the disc margin, where it is expressed prior to the onset of eye patterning, impedes growth of the disc and prevents all aspects of MF initiation. Furthermore, we show that the activation of the $\mathrm{Hh}$ signaling pathway is sufficient to induce ommatidial development in the absence of wg and dpp function.

The results presented here conflict with previous reports suggesting that $\mathrm{Hh}$ is not required for the M $\mathrm{F}$ initiation (Heberlein et al. 1993; Ma et al. 1993; Jarman et al. 1995). This notion was based on the following evidence. MF initiation is normal in the hypomorphic $\mathrm{hh}^{1}$ allele (Heberlein et al. 1993) and in ato mutants (Jarman et al. 1995) where hh is not expressed in the developing photoreceptors. We show that hh is al ready present al ong the margin of the late second/ early third instar disc prior to ato expression and that MF initiation is dependent on $\mathrm{Hh}$ function in the disc margin. Furthermore, we show that ato expression is strictly dependent on $\mathrm{Hh}$ input. Therefore, by genetic criteria, $\mathrm{hh}^{1}$ is a partial loss-offunction allele. It is likely that MF initiation in $\mathrm{hh}^{1} \mathrm{mu}-$ tants initiates normally because the early expression of $\mathrm{hh}$ in the margin, as hh expression in other imaginal discs, is normal in the $\mathrm{hh}^{1}$ mutant. Temperature-shift experiments performed with the $\mathrm{hh}^{\mathrm{ts}}$ allele at the early third instar stage did not prevent initiation of the MF, presumably because the requirement for $\mathrm{Hh}$ begins already at an earlier stage. Because of the perdurance of the $\beta$-galactosi dase protein used to analyze hh expression in the second instar, we cannot accurately determine the onset of hh expression in the disc margin, and hence, its time of action. Although we cannot exclude the possibility that $\mathrm{Hh}$ function begins at an earlier stage than the onset of ato expression, we believe that Hh's function is also involved later in the initiation of the M F. Clones of pka induced at the second instar larval stage still express ato and generate ectopic M Fs (Strutt et al. 1995; Pan and Rubin 1995; M. Domínguez and E. Hafen unpubl.). Similarly, late-induced pka wg dpp clones still induce ectopic ato expression, supporting the view that cells must receive a direct input from $\mathrm{Hh}$ to initiate the MF. In vertebrates, inhibition of protein kinase A phenocopies the effects of ectopic expressi on of vertebrate $\mathrm{Hh}$ proteins in the devel oping eye (Hammerschmidt et al . 1996) suggesting that the mechanisms of the $\mathrm{Hh}$ signaling is conserved.

The early function of $\mathrm{Hh}$ in Drosophila eye development described here may have parallels during vertebrate eye development. In the Zebrafi sh embryo, two hh genes are expressed in the floor of the diencephal on adjacent to the devel oping optic vesicles that express Pax2 adjacent to the $\mathrm{Hh}$ domain and Pax6 in the presumptive distal portion far from the source of $\mathrm{Hh}$ (Ekker et al. 1995). High levels of Sonic hedgehog (SHh) reduce Pax6 expression and result in the expansion of Pax2 expression. A similar regulatory relationship between Shh and Pax6 has recently been shown in the developing neural tube (Ericson et al . 1997). Hence, it is possible that one of the early functions of $\mathrm{Hh}$ during Drosophila eye development is the regulation of eyeless, the Pax6 homol og in Drosophila. Consistent with this notion is the fact that the early expression we detect in second instar eye imaginal discs is confined to the region surrounding the eye field.

In contrast to the direct role of $\mathrm{Hh}$ in $\mathrm{MF}$ initiation, it appears that the control of MF initiation by Dpp is indirect; it acts by repressing $w g$. The negative regulation of wg by Dpp at the disc margins (Wiersdorff et al. 1996; Chanut and Heberlein 1997; Pignoni and Zipursky 1997) provides an explanation for why ectopic dpp-expressing cells can only initiate an ectopic MF from the anterior margin (Chanut and Heberlein 1997; Pignoni and Zipursky 1997), where wg is normally expressed (M a and Moses 1995; Hesl ip et al. 1997). The ectopic M Fs induced by $\mathrm{Dpp}$ at the anterior margin are likely initiated by $\mathrm{Hh}$, which is al so expressed at the dorsal margin in theyoung eye disc (Fig. 3B,C,G). A role for $\mathrm{Hh}$ in MF initiation at the anterior disc margin caused by temporally removing wg function is supported by the observation that this phenotype is suppressed by hh (Treisman and Rubin 1995).

A striking phenotype is the formation of ectopic eye fields in the vicinity of marginal Mad (Wiersdorff et al. 
1996) and tkv strll (Fig. 2H) clones. This dramatic reorganization of the eye disc resembles the production of ectopic appendages as a consequence of local loss of Dpp reception at the compartment boundary in the leg discs (Brook and Cohen 1996; Jiang and Struhl 1996; Johnston and Schubiger 1996; Morimura et al. 1996; Peton and Hoffmann 1996; Theisen et al. 1996). These ectopic appendages appear to result from the novel juxtaposition of dpp-expressing cells and cells expressing wg because of their failure to respond to Dpp. A similar mechanism could account for the formation of ectopic eye fields in mosai c Mad and tkv eye discs because it has been shown that wg expression is activated in Mad mutant clones and in partial loss-of-function mutants of dpp in the eye (Wiersdorff et al. 1996; Chanut and Herberlein 1997).

Like in the leg disc, the early expression of dpp and wg in the disc margins may be induced by Hh. Several lines of evidence suggest that this is the case. First, hh is expressed at the disc margin of the second instar disc and its expression overlaps with the domain of wg and dpp at this stage. Second, removal of pka, which mimics the effects of ectopic $\mathrm{Hh}$ expression, results in ectopic expression of dpp or of wg. Like in the antenna and leg disc, the response to the removal of pka is limited to cells anterior to the hh-expressing cells, and differs in different regions of the eye disc. In the anterior margin of the eye disc, loss of pka induces expression of wglike in the ventral domain of the leg and the antenna. In the internal part of the eye disc, loss of pka induces dpp expression like the dorsal region of the leg and antenna. Furthermore, the repression of wg by Dpp occurs not only in the margin but also in eye internal cells lacking pka. It is, therefore, likely that $\mathrm{Hh}$ directly induces early expression of wg and dpp by antagonizing pka activity at the eye disc margin. Thus, the regulatory relationship between Hh, Dpp, and Wg may be similar in the eye disc and the leg disc despite obvious differences in the way the discs develop. Patterning of the leg is controlled by the juxtaposition of two clonally unrelated cell populations, anterior and posterior cells, and is not linked to differentiation (Basler and Struhl 1994; Brook and Cohen 1996; Jiang and Struhl 1996). The eye disc, however, develops by a lineage-independent mechanism and differentiation is a prerequisite for progression of the patterning process.

Different thresholds for $\mathrm{Hh}$ activity to induce ommatidial assembly

Our analysis of hh in the eye disc has shown that there are different requirements for $\mathrm{Hh}$ activity at the disc margins and in the internal part of the eye disc, as observed by the different degree on nonautonomy between hh internal and hh marginal clones. Loss of hh activity in the internal region of the disc has very little consequences, presumably because the presence of $\mathrm{hh}^{+}$tissue is sufficient to rescue the lack of hh function over a relatively long distance (about three ommatidial units). In contrast, the loss of hh expression at the margin, even in small clones, is not completely rescued by the adjacent $\mathrm{Hh}$-producing ommatidia. As the M F progresses in a mosaic disc carrying a marginal hh clone, only a single ommatidial unit adjacent to $\mathrm{Hh}$-secreting ommatidia is rescued. Thus, there appears to be an essential requirement for hh function at the disc margin to induce ommatidial differentiation in the internal cells. There is no detectable hh expression in the disc margins at the time of MF progression in the late third instar larval disc (Ma et al. 1993; M. Domínguez and E. Hafen unpubl.). Thus, this requirement for Hh may be related to its early expression in the disc margin. In the absence of early marginal expression of hh, the initiation of ommatidial development appears to require a higher concentration of $\mathrm{Hh}$ so that only the mutant cells at the boundary to the $\mathrm{Hh}$-secreting cells receive sufficient $\mathrm{Hh}$ to be rescued. The early expression of hh at the margin would result in a gradient of $\mathrm{Hh}$ activity toward the center of the eye disc, predisposing cells in the presumptive eye field to become ommatidial cells later. In support of this, we observed that expression of dpp-lacZ and of ptc-lacZ are graded from the margin toward the center of the disc. The graded expression of $\mathrm{dpp}$ becomes uniform and expands throughout the posterior part of the disc upon ectopic expression of hh in the second instar larval stage (Pignoni and Zipursky 1997). It is interesting to note that in $\mathrm{hh}^{1}$ eye discs, where no $\mathrm{Hh}$ protein is detected during the mid to late third instar stage (Huang and Kunes 1996) the MF progression stops first in the center of the disc and only later near the disc margin (Heberlein et al. 1993). This observation is consistent with the notion that in the wild type, central cells farthest away from the disc margin require higher $\mathrm{Hh}$ levels to initiate neural development than cells located near the margins.

A similar early and late requirement of $\mathrm{Hh}$ signaling as proposed here for eye patterning has recently been shown for the differentiation of motor neurons in the vertebrate neural tube (Ericson et al. 1996). In this system, early expression of $\mathrm{SHh}$ in the notochord is important to induce a ventralized state in the cells of the neural tube. This ventralized state is marked by the repression of pax 7 and pax 3 expression in the ventral neural tube and is required for later specification of motor neurons by $\mathrm{Hh}$ secreted from floor-plate cells. In each case, the same cells, ommatidial precursor cells or motor neuron precursors, require for their differentiation distinct phases of $\mathrm{SHh}$ signaling originating from different cell populations: marginal cells and differentiating photoreceptor cells in the eye disc and notochord cells and floor plate cells in the neural tube. We note that the reiterative use of the $\mathrm{Hh}$ signaling pathway during patterning of the Drosophila eye and the differentiation within the vertebrate neural tube is similar to the reiterative use of the Ras/MAP kinase pathway during the specification of the different cell types in the developing eye (Freeman 1996). Hh signal ing in the Drosophila eye may thus be another example of how the same signaling pathway is used repeatedly to advance the devel opmental state of cells and tissues in a ratchetlike manner. 


\section{Materials and methods}

Fly stocks

The hh allele used, $\mathrm{hh}^{\mathrm{AC}}$, is a null allele that is a small del etion of sequences of the hh promoter and part of the coding region (Ma et al. 1993). In genetic clones, $\mathrm{hh}^{\mathrm{AC}}$ behaves as other null alleles ( $\mathrm{Ma}$ et al. 1993; Basler and Struhl 1994). dpp ${ }^{\mathrm{H} 61}$ and $\mathrm{wg}^{\mathrm{CX} 4}$ are null alleles. dpp ${ }^{\mathrm{d} 12}$ and $\mathrm{pka}^{\mathrm{DCO}-\mathrm{B3}}$ are strong loss-offunction alleles (described in Jiang and Struhl 1995; Li et al. 1995). The enhancer-trap strain $\mathrm{hh}^{\mathrm{P} 30}$ ( $\mathrm{M}$ a et al. 1993) was used to monitor expression of the hh gene. ptc expression was monitored by use of the ptc ${ }^{\mathrm{AT} 96}$ enhancer-trap line (kindly provided by $\mathrm{G}$. Struhl). In the eye disc, Ptc protein is detected at low levels in all anterior cells and at higher levels in cells in the M F and in some cells posterior to the MF (Strutt and M lodzik 1996). ptc-lacZ expression in the ptc ${ }^{\text {AT96 }}$ enhancer-trap line is high in cells posterior to the MF. dpp expression was monitored with a BS3.0 reporter construct (Blackman et al. 1991) inserted on the second chromosome. This reporter line reproduces the expression of the endogenous dpp gene (M asucci et al. 1990).

\section{Somatic clones}

Mitotic recombination clones were generated by use of the FRT-FLP technique (Xu and Rubin 1993) al one or in combination with the Minute technique (M orata and Ripoll 1975) to give the mutant clone a growth advantage. The genotype of the larvae in Figure 1 and $2 \mathrm{~A}-\mathrm{C}$ are y w hs-FLP122; $\mathrm{FRT}^{82 \mathrm{~B}} \mathrm{hh}^{\mathrm{AC}}$ / $\mathrm{FRT}^{82 \mathrm{~B}}$ arm-lacZ. In these experiments, the $\mathrm{hh}^{\mathrm{AC}} / \mathrm{hh}^{\mathrm{AC}}$ homozygous mutant tissue and the twin spot $\left(\mathrm{hh}^{+}\right.$arm-lacZ/hh+ arm-lacZ) are marked, respectively, by the absence or the increased levels of lacZ staining in relation to the heterozygous tissue. The genotype of discs shown in Figure $2 \mathrm{E}-\mathrm{G}$ is y w hsFLP122; $\mathrm{FRT}^{82 \mathrm{~B}} \mathrm{hh}^{\mathrm{AC}} / \mathrm{FRT}^{82 \mathrm{~B}} \mathrm{M}(3 \mathrm{R})^{67 \mathrm{C}}$. The genotype of the disc shown in Figure $2 \mathrm{H}$ is y w hs-FLP122; tkv ${ }^{\text {strll }} \mathrm{FRT}^{40}$ / $\mathrm{M}(2)^{25 \mathrm{~A}} \mathrm{FRT}^{40}$. The tkv ${ }^{\text {strll }}$ allele (provided by $\mathrm{K}$. Basler) is an amorphic allele, and at the margin, but not in the internal region, of the eye disc only very small clones are recovered. To generate larger clones of $\mathrm{tkv}^{\text {strll }}$ at the margin, the somatic clones were induced in a Minute background. To compare the phenotype of loss of $h$ h and loss of Dpp-reception, clones of cells

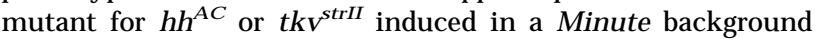
were generated. Eye-antennal discs with supernumerary eye field and duplicated antenna were obtained only in discs of the genotype $t \mathrm{k}^{\text {strll }} \mathrm{M}^{+}$. In addition, we found that mosaic discs of the genotype $h^{A C} M^{+}$showed signs of cessation of the M F, such as mature ommatidia in the leading front of Elav expression, and absence of dpp-lacZ expression. These phenotypes presumably result from the loss of hh in a large internal area.

The genotype of the discs carrying triple mutant clones shown in Figure $4 \mathrm{~A}$ is $\mathrm{pka}^{\mathrm{DCO}-\mathrm{B} 3}$ stc $\mathrm{FRT}^{40} / \mathrm{arm}-\mathrm{lacZ}, \mathrm{FRT}^{40}$ in Figure $4, \mathrm{~B}$ and $\mathrm{C}$ is $\mathrm{dpp}^{\mathrm{H} 61} \mathrm{wg}^{\mathrm{CX} 4} \mathrm{pka}^{\mathrm{DCO}-\mathrm{B3}}$ stc $\mathrm{FRT}^{39 \mathrm{E}} /$ $\mathrm{Dp}(2 ; 2) \mathrm{VT} 1\left(\mathrm{dpp}^{+}\right) \mathrm{Dp}(1 ; 2) \mathrm{sc}^{19}\left(\mathrm{y}^{+}\right) \mathrm{FRT}^{39 E}$ hs-Flp, in Figure 4D is y hs-Flp122/+; wg ${ }^{\mathrm{CX} 4} \mathrm{pka}^{\mathrm{DCO}-\mathrm{B3}} \mathrm{FRT}^{40} / \mathrm{arm}-\mathrm{lacZ} \mathrm{FRT}^{40}$, and in Figure 4, E and F, is y hs-Flp122/ +; dpp ${ }^{d 12}$ pka $^{\text {DCO-B3 }} \mathrm{FRT}^{40}$ / arm-lacZ $\mathrm{FRT}^{40}$. In all cases, the FLP gene was activated in first instar larvae by heat shocking the larvae for $1 \mathrm{hr}$ at $38^{\circ} \mathrm{C}$. Wandering third instar eye discs were dissected for histochemistry.

\section{Immunofluorescence staining}

Eye imaginal discs from second to third instar larvae were dissected, stained, and analyzed as described by Gaul et al. (1992). A rat monoclonal anti-Elav antibody diluted 1:50, a rabbit polyclonal anti-Ato antibody diluted 1:2000, a rabbit polyclonal anti- $\beta$-gal actosi dase antibody (Cappel) diluted 1:400 or a mouse monoclonal anti- $\beta$-galactosidase antibody (Cappel) diluted $1: 2000$ were used. In the experiments in which loss of Elav staining was used to mark the clones indirectly, discs were double-stained with a mouse anti-Arm antibody (N2 7A1 Armadillo) from the Hybridoma Bank. Arm protein accumulates in the adherens junctions and becomes concentrated around the apical tips of cells in the MF and in photoreceptor cells as they gather into clusters. Modulation of Arm protein was used as morphological criterion for the differentiation stage of cells in the eye. Secondary antibodies, either a FITC- or a Texas-Redconjugated, were from Jackson Inc. Eye discs were incubated overnight with a mixture of the two primary antibodies, washed several times and then incubated for $2 \mathrm{hr}$ in a mixture of the two secondary antibodies.

\section{Acknowledgments}

We thank K. Dücker and J. Riesgo for discussions and D. Gubb, J.F. de Celis, M. Freeman, P. Lawrence, and K. Basler for critical comments of the manuscript. Wethank K. M oses, K. Basler, and G. Struhl for fly stocks, Y. Jan for the Ato antibody. Part of this work was carried out in Dr. P. Lawrence's laboratory. M.D. was supported by a postdoctoral fellowship from Human Frontiers Science Program and is presently supported by a postdoctoral fellowship from European Molecular Biology Organization (EMBO). E.H. acknowledges the support from the Swiss $\mathrm{Na}$ tional Science Foundation.

The publication costs of this article were defrayed in part by payment of page charges. This article must therefore be hereby marked "advertisement" in accordance with 18 USC section 1734 solely to indicate this fact.

\section{References}

Basler, K. and G. Struhl. 1994. Compartment boundaries and the control of Drosophila limb pattern by hedgehog protein. Nature 368: 208-214.

Blackman, R.K., M. Sanicola, L.A. Raftery, T. Gillevet, and W.M. Gelbart. 1991. An extensive 3' cis-regulatory region directs the imaginal disk expression of decapentaplegic, a member of theTGF-beta family in Drosophila. Devel opment 111: 657-666.

Bonini, N.M. and K.W. Choi. 1995. Early decisions in Drosophila eye morphogenesis. Curr. O pin. Genet. Dev. 5: 507515.

Bokor, P. and S. DiN ardo. 1996. The roles of hedgehog, wingless, and lines in patterning of the dorsal epidermis in Drosophila. Development 122: 1083-1092.

Brook, W.J. and S.M. Cohen. 1996. Antagonistic interactions between Wingless and Decapentaplegic responsible for dorsal-ventral pattern in the Drosophila leg. Science 273: 13731377.

Burke, R. and K. Basler. 1996. Hedgehog dependent patterning in the Drosophila eye can occur in the absence of Dpp signaling. Dev. Biol. 179: 360-368.

Capdevila, J., M .P. Estrada, H.E. Sánchez, and I. Guerrero. 1994. The Drosophila segment polarity gene patched interacts with decapentaplegic in wing devel opment. EMBO J. 13: 7182.

Chanut, F. and U. Heberlein. 1997. Role of decapentaplegic in initiation and progression of the morphogenetic furrow in the developing Drosophila retina. Development 124: 559567.

Ekker, S.C., A.R. Ungar, P. Greenstein, D.P. von Kessler, J.A. Porter, R.T. M oon, and P.A. Beachy. 1995. Patterning activi- 
ties of vertebrate hedgehog proteins in the developing eye and brain. Curr. Biol. 5: 947-955.

Ericson, J., S. M orton, A. Kawakami, H. Roelink, and T.M. Jessell. 1996. Two critical periods of sonic hedgehog signaling required for the specification of motor neuron identity. Cell 87: 661-674.

Ericson, J., P. Rashbass, A. Schedl, S. Brennermorton, A. Kawakami, V. Vanheyningen, T.M. Jessell, and J. Briscoe. 1997. Pax 6 controls progenitor cell identity and neuronal fate in response to graded shh signaling. Cell 90: 169-180.

Forbes, A.J., Y. N akano, A.M. Taylor, and P.W. Ingham. 1993. Genetic analysis of hedgehog signalling in the Drosophila embryo. Development 119: 115-124.

Freeman, M. 1996. Reiterative use of the EGF receptor triggers differentiation of all cell types in the Drosophila eye. Cell 87: $651-660$.

Gaul, U., G. Mardon, and G.M. Rubin. 1992. A putative Ras GTPase activating protein acts as a negative regulator of signaling by the sevenless receptor tyrosine kinase. Cell 68: 1007-1019.

Gómez-Skarmeta, J.L. and J. M odolell. 1996. Araucan and caupolican provide a link between compartment subdivisions and patterning of sensory organs and veins in the Drosophila wing. Genes \& Dev. 10: 2935-2945.

Hammerschmidt, M., M.J. Bitgood, and A.P. McM ahon. 1996. Protein kinase $A$ is a common negative regulator of hedgehog signaling in the vertebrate embryo. Genes \& Dev. 10: $647-658$.

Hammerschmidt, M., A. Brook, and A.P. McM ahon. 1997. The world according to hedgehog. Trends Genet. 13: 14-21.

Heberlein, U. and K. Moses. 1995. M echanisms of Drosophila retinal morphogenesis: The virtues of being progressive. Cell 81: 987-90.

Heberlein, U., T. Wolff, and G.M. Rubin. 1993. The TGF $\beta$ homolog dpp and the segment polarity gene hedgehog are required for propagation of a morphogenetic wave in the Drosophila retina. Cell 75: 913-926.

Heberlein, U., C.M. Singh, A.Y. Luk, and T.J. Donohoe. 1995. Growth and differentiation in the Drosophila eye coordinated by hedgehog. Nature 373: 709-711.

Heslip, T.R., H. Theisen, H. Walker, and L. M arsh. 1997. Shaggy and Dishevelled exert opposite effects on wingless and de capentaplegic expression and on positional identity in imaginal discs. Development 124: 1069-1078.

Huang, Z. and S. Kunes. 1996. Hedgehog, transmitted along retinal axons, triggers neurogenesis in the devel oping visual centers of the Drosophila brain. Cell 86: 411-422.

Jarman, A.P., E.H. Grell, L. Ackerman, L.Y. Jan, and Y.N. Jan. 1994. atonal is the proneural gene for Drosophila photoreceptors. Nature 369: 398-400.

Jarman, A.P., Y. Sun, L.Y. Jan, and Y.N. Jan. 1995. Role of the proneural gene, atonal, in formation of Drosophila chordotonal organs and photoreceptors. Devel opment 121: 20192030.

Jiang, J. and G. Struhl. 1995. Protein kinase A and hedgehog signaling in Drosophila limb devel opment. Cell 80: 563-572.

- - - 1996. Compl ementary and mutually exclusi ve activities of decapentaplegic and wingless organise axial patterning during Drosophila leg development. Cell 86: 401-409.

Johnston, L.A. and G. Schubiger. 1996. Ectopic expression of wingless in imaginal discs interferes with decapentaplegic expression and alters cell determination. Development 122: 3519-3529.

Johnson, R.L., J.K. Grenier, and M .P. Scott. 1995. patched overexpression alters wing disc size and pattern: Transcriptional and post-transcriptional effects on hedgehog targets. Devel- opment 121: 4161-4170.

Lee, J.J., K.D. Von, S. Parks, and P.A. Beachy. 1992. Secretion and localized transcription suggest a role in positional signaling for products of the segmentation gene hedgehog. Cell 71: 33-50.

Lepage, T., S.M. Cohen, F.J. Díaz-Benjumea, and S.M. Parkhurst. 1995. Signal transduction by CAMP-dependent protein kinase $A$ in Drosophila limb patterning. Nature 373: 711-715.

Li, W., J.T. Ohlmeyer, M.E. Lane, and D. Kalderon. 1995. Function of protein kinase $A$ in hedgehog signal transduction and Drosophila imaginal disc development. Cell 80: 553-562.

Lindsley, D.L. and G.G. Zimm. 1992. The genome of Drosophila melanogaster. Academic Press, San Diego, CA.

$\mathrm{Ma}, \mathrm{C}$. and K. Moses. 1995. wingless and patched are negative regulators of the morphogenetic furrow and can affect tissue polarity in the devel oping Drosophila compound eye. Development 121: 2279-2289.

Ma, C., Y. Zhou, P.A. Beachy, and K. M oses. 1993. The segment polarity gene hedgehog is required for progression of the morphogenetic furrow in the developing Drosophila eye. Cell 75: 927-938.

M asucci, J.D., R.J. M iltenberger, and F.M . Hoffmann. 1990. Pattern-specific expression of the Drosophila decapentaplegic gene in imaginal disks is regulated by 3 ' cis-regulatory elements. Genes \& Dev. 4: 2011-2023.

M orata, G. and P. Ripoll. 1975. Minutes: Mutants of Drosophila autonomously affecting cell division rate. Dev. Biol. 42: 211-221.

Morimura, S., L. Maves, Y. Chen, and F.M. Hoffmann. 1996. decapentaplegic overexpression affects Drosophila wing and leg imaginal disc devel opment and wingl ess expression. Dev. Biol. 177: 136-151.

Mullor, J.L., M. Calleja, J. Capdevilla, and I. Guerrero. 1997. Hedgehog activity, independent of Decapentaplegic, participates in wing disc patterning. Devel opment 124: 1227-1237.

N g, M., F.J. Diaz-Benjumea, J. P. Vincent, J. Wu, and S.M. Cohen. 1996. Specification of the wing by localized expression of wingless protein. Nature 381: 316-318.

Pan, D. and G.M. Rubin. 1995. CAM P-dependent protein kinase and hedgehog act antagonistically in regulating decapentaplegic transcription in Drosophila imaginal discs. Cell 80: 543-552.

Peton, A. and M. Hoffmann. 1996. Decapentapl egic restricts the domain of wingless during Drosophila limb patterning. Nature 382: 162-165.

Peton, A., S.B. Selleck, and M. Hoffmann. 1997. Regulation of cell cycle synchronization by decapentaplegic during Drosophila eye devel opment. Science 275: 203-206.

Pignoni, F. and L. Zipursky. 1997. Induction of Drosophila eye development by Decapentaplegic. Development 124: 271278.

Strutt, D.I. and M. Mlodzik. 1995. Ommatidial polarity in the Drosophila eye is determined by the direction of furrow progression and local interactions. Development 121: 4247-4256.

- - - 1996. The regulation of hedgehog and decapentaplegic during Drosophila eye imaginal disc development. Mech. Dev. 58: 39-50.

Strutt, D.I., V. Wiersdorff, and M. M lodzik. 1995. Regulation of furrow progression in the Drosophila eye by CAMP-dependent protein kinase A. Nature 373: 705-709.

Tabata, T., S. Eaton, and T.B. Kornberg. 1992. The Drosophila hedgehog gene is expressed specifically in posterior compartment cells and is a target of engrailed regulation. Genes \& Dev. 6: 2635-2645. 


\section{Domínguez and Hafen}

Tabata, T., C. Schwartz, E. Gustavson, Z. Ali, and T.B. Kornberg. 1995. Creating a Drosophila wing de novo, the role of engrailed, and the compartment border hypothesis. Development 121: 3359-3369.

Theisen, H., J. Purcell, M. Bennett, D. Kansagara, A. Syed, and J.L. Marsh. 1996. Developmental territories created by mutual antagonism between WG and DPP. Development 122: 3939-3948.

Treisman, J. and G.M. Rubin. 1995. wingless inhibits morphogenetic furrow movement in the Drosophila eye disc. Development 121: 3519-3527.

Wehrli, M. and A. Tomlinson. 1995. Epithelial planar polarity in the developing Drosophila eye. Development 121: 24512459.

Wiersdorff, V., T. Lecuit, S.M. Cohen, and M. Mlodzik. 1996. Mad acts downstream of D pp receptors, revealing a differential requirement for dpp signaling in initiation and propagation of morphogenesis in the Drosophila eye. Development 122: 2153-2162.

Wolff, T. and D.F. Ready. 1993. Pattern formation in the Drosophila retina. In The development of Drosophila melanogaster (ed. M. Bate and A. Martinez-Arias), pp. 1277-1326. Cold Spring Harbor Laboratory Press, Cold Spring Harbor, NY.

Xu, T. and G.M. Rubin. 1993. Analysis of genetic mosaics in developing and adult Drosophila tissues. Development 117: 1223-1237. 


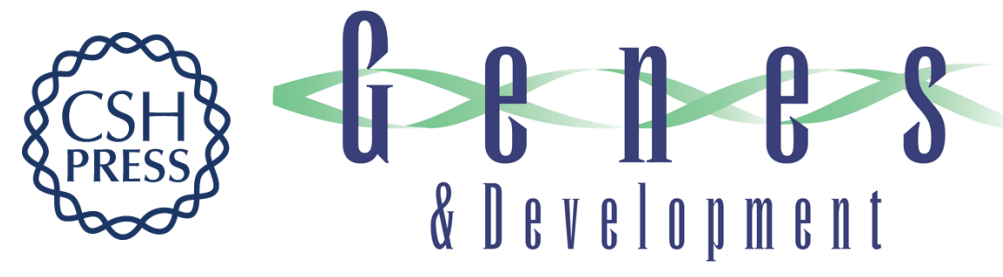

\section{Hedgehog directly controls initiation and propagation of retinal differentiation in the Drosophila eye}

María Domínguez and Ernst Hafen

Genes Dev. 1997, 11:

Access the most recent version at doi:10.1101/gad.11.23.3254

\section{License}

Email Alerting

Receive free email alerts when new articles cite this article - sign up in the box at the top

Service right corner of the article or click here.

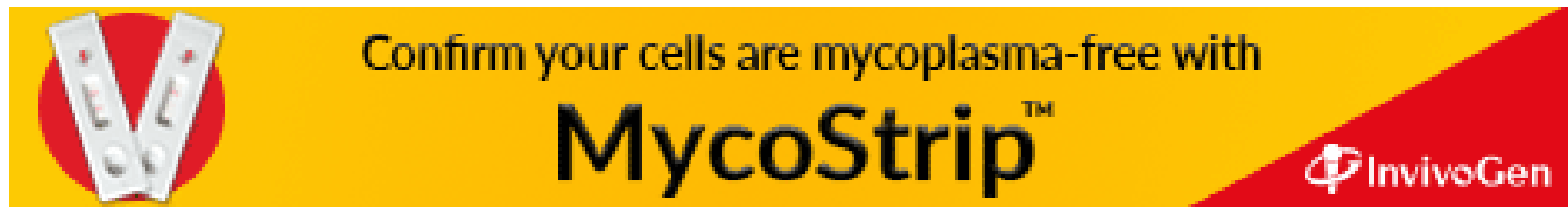

